\title{
A Survey on Terrain Based Navigation for AUVs
}

\author{
Sebastian Carreño, ${ }^{*}$ Pere Ridao, ${ }^{* *}$ Philip Wilson, ${ }^{*}$ \\ and Yvan Petillot*** \\ * School of Engineering Sciences, University of Southampton, SO17 \\ 1BJ, United Kingdom (e-mail: \{sc3v09,philip.wilson\}@soton.co.uk) \\ ** Department of Computer Engineering, University of Girona, 17071, \\ Spain (e-mail: pere@eia.udg.edu) \\ *** Oceans Systems Laboratory, Heriot-Watt University, EH14 4 AS, \\ UK(e-mail: y.r.petillot@hw.ac.uk)
}

\begin{abstract}
:
Terrain Based Navigation (TBN) is a method rooted to the early cruise missile navigation systems, when GPS was not yet available. For decades, TBN has been applied as a complementary system to INS navigation for Unmanned Aerial Vehicles (UAV). In the field of Autonomous Underwater Vehicles (AUVs), it has the potential to bound the drift inherent to dead reckoning navigation, based on INS and/or Doppler Velocity Log (DVL) sensors, as well as to make the navigation beyond the areas of coverture of the acoustic transponder networks, a reality. This paper overviews the main concepts related to TBN and present an exhaustive survey of the works reported in the literature. As a main contribution, a table comparing the motion and the measurement models, as well as the probabilistic framework used for the estimation is reported. An effort has been put on unifying the diverse nomenclature used across the surveyed works. We aim this paper to become an starting point for the researchers interested in this technology, with pointers to the most interested works in the area.
\end{abstract}

Keywords: Aided navigation, surveys, autonomous vehicles, localization, underwater vehicles.

\section{INTRODUCTION}

The Terrain Based Navigation (TBN) problem, also named terrain aided navigation (TAN) is the name used by the marine and aerial robotics communities to refer to the more general problem of mobile robot localization with a known map. In particular, TBN solves for the robot pose given an a priori known map, fusing information from dead reckoning navigation with map referenced observations. The principle is the same as has been used for centuries: Localize the vehicle based on observations of known characteristics of the map.

TBN has been mainly applied to aerial and underwater vehicles. During the last years the accuracy and extension of the maps has been increased considerably, and TBN has been adopted as a method to complement INS navigation, as an alternative to when GPS is not available. In other fields of application, like underwater, TBN is still at a research stage, not having evolved into a commercial product. During the last two decades, the scientific community working with mobile robots, has pushed forward the boundaries of the knowledge facing an even more challenger problem: the Simultaneous Localization and Mapping ( see Durrant-Whyte and Bailey (2006)). Recently, these techniques have start to be slowly adapted to underwater environments (see Mallios et al. (2009)).

\footnotetext{
^ This work was supported by the FREE ${ }_{s u b}$ NET project.
}

Both SLAM and TBN have a great potential to improve the autonomy of the underwater vehicles, allowing AUVs to freely move abroad the areas of coverage of the acoustic transponder networks.

In this paper we focus on the review of the TBN techniques considering SLAM out of scope of this work. Although our interest AUV navigation, because TBN is a mature technique for aerials vehicles, representative works of this area hav been included in our study. The paper is organized as follows: First the problem of the autonomous navigation is presented, then, in chapter two, the TBN problem is solved in a general form for UUV and AUV, the seminal work of TERCOM is described in chapter three, followed by a selection of the most representative Bayesian estimation TBN techniques carried out so far, grouped by their main filters in chapter four, to complement this survey a equations table comparing the main components of each technique presented so far is provided, to finalize some conclusions are presented.

\section{THE TERRAIN BASED NAVIGATION PROBLEM}

Using navigation sensors it is possible to measure the robot velocity, acceleration and attitude, to be used as the input for the dead reckoning equations needed to compute the robot pose. Due to the noisy measurements and the position estimate will rapidly grow without bounds. In some applications GPS can be used to provide absolute fixes to 
bound the drift. However other domains like underwater, covert operations, or out space applications the GPS is not available. Absolute positioning fixes can be provided underwater by acoustic positioning like the Long Baseline System (LBL), the Short Baseline (SBL), the Ultra Short Baseline (USBL) or the GPS eqquiped intelligent Buoys (GIB). However, these systems require time for deployment. Moreover they constrain the vehicle operation to a certain area of coverage. TBN has a potential to become an alternative to satellite navigation and acoustic transporter networks.

TBN takes advantage of existing digital terrain maps of target area, where the vehicle shall navigate. Conventional dead reckoning navigation methods provide an a prior estimate of the robot pose within the map. Then, using exteropceptive sensors, terrain observations are obtained to be correlated to the a priori known map in order to compute the robot pose.

\subsection{Problem statement}

Let

- $\{\mathbb{E}\}$ be a inertial earth-fixed frame.

- $\{\mathrm{B}\}$ be the robot fixed frame.

- $\eta_{t}=[x y z \phi \theta \psi]$ be the robot pose at time $t$ referenced to $\{\mathbb{E}\}$.

- $v_{t}=[u v w p q r]$ be the robot velocity referenced to $\{\mathrm{B}\}$.

- Let $\operatorname{IM}(\eta)$ be a digital elevation map of the environment, assumed to be known a priori.

- $\mathbf{x}_{t}$ be the state vector, usually containing the robot pose $\eta_{t}$ or its bias $\varepsilon_{\eta_{t}}$.

- $\mathbf{z}_{t}$ be the vector of observations coming from exteroceptive sensors like:

- $s_{t, i}$ : range measurements at bearing $i$ measured from the radar, the sonar altimeter $(i=0)$, or the multibeam sonar profiler $(0 \leq i \leq N)$.

- $r_{t, i}$ : projection of the range measurement $s_{t, i}$ on the $z$ vertical axis of the $\mathbb{E}$-frame. For the sake of simplicity the origins of the sensor and the robot frames are chosen to be coincident.

- $d_{t}$ : the depth of the UUV measured with respect to the sea level, usually provided by a pressure sensor.

- $a_{t}$ : the altitude of an aerial vehicle measured with respect to the sea level, usually provided by a pressure sensor.

Then the TBN consist on estimating the robot velocity $v_{t}$, referenced to the vehicle $\mathrm{B}$-frame, and/or the robot pose $\eta_{t}$, referenced to the inertial $\mathbb{E}$-frame, by matching the measured depth $d_{t}$ (altitude $a_{t}$ for UAVs) and ranges $s_{t, i}$ with the terrain elevation map stored in $\mathrm{M}$.

\subsection{Motion model}

A nonlinear kinematics motion model can be used to relate the robot velocity expressed in the $\mathbb{B}$-frame to the robot pose referenced to the $\mathbb{E}$-frame:

$$
\dot{\mathbf{x}}=J(\eta) v ; J(\eta)=\operatorname{diag}\left\{R(\eta) ; J_{2}(\eta)\right\}
$$

where $R(\eta)$ is the Roll, Pitch and Yaw rotation matrix and $J_{2}(\eta)$ (see Fossen (2002)) is the velocity transformation matrix, which translates the B-referenced angular velocity vector into the Euler angles derivatives. For AUVs, the linear velocity is commonly provided by a Doppler Velocity Log (DVL) sensor, while the angular velocity is commonly provided from Fiber Optical (FOG) or Ring Laser Gyros (RLG).

\subsection{Measurement model}

For an AUV (Figure 1) the measurement model is given by:

$$
r_{t, i}=\mathbb{M}_{i}\left(\eta_{t}\right)-d_{t}+\mathbf{v}_{t}, \forall 0 \leq i \leq N
$$

where $\mathbf{v}_{t}$ is the measurement noise and $N=0$ when a sonar altimeter is used. If a multi-beam sonar profiler is used, $N$ is the number of beams. When a vertical sonar altimeter is used, $r_{t, i}=s_{t, i}$. It is worth noting that $\mathrm{M}_{i}\left(\eta_{t}\right)$ is the map elevation at the point where the sonar $i$ beam intersects the terrain surface. For an aerial vehicle (Figure 2), the equation becomes:

$$
s_{t}=a_{t}-\mathrm{M}\left(\eta_{t}\right)+\mathbf{v}_{t}
$$

Note that in both cases the map $\mathrm{M}$, also known as terrain function, is a nonlinear function of $\eta_{t}$. Then, the nonlinear observation equation can be formulated as follows:

$$
\begin{gathered}
\mathbf{z}_{t}=h\left(\mathbf{x}_{t}\right)+\mathbf{v}_{t} ; \\
\left.h\left(\mathbf{x}_{t}\right)=\left[h_{(} \mathbf{x}_{t}\right) \ldots h_{N}\left(\mathbf{x}_{t}\right)\right]^{T} \\
h_{i}\left(\mathbf{x}_{t}\right)=\mathbb{M}_{i}\left(\eta_{x y_{t}}\right)-\eta_{z_{t}}+\mathbf{v}_{t}
\end{gathered}
$$

where $\eta_{z_{t}}=d_{k}$ and $\mathbf{z}_{t}$ is a vector containing the ground clearance measurements corresponding to each sonar beam, and $\mathbf{x}_{t}$ contains at least the robot pose:

$$
\mathbf{z}_{t}=\left[r_{t, 0} \ldots r_{t, N}\right]^{T} ; \mathbf{x}_{t}=\left[\eta_{t} \ldots\right]^{T}
$$

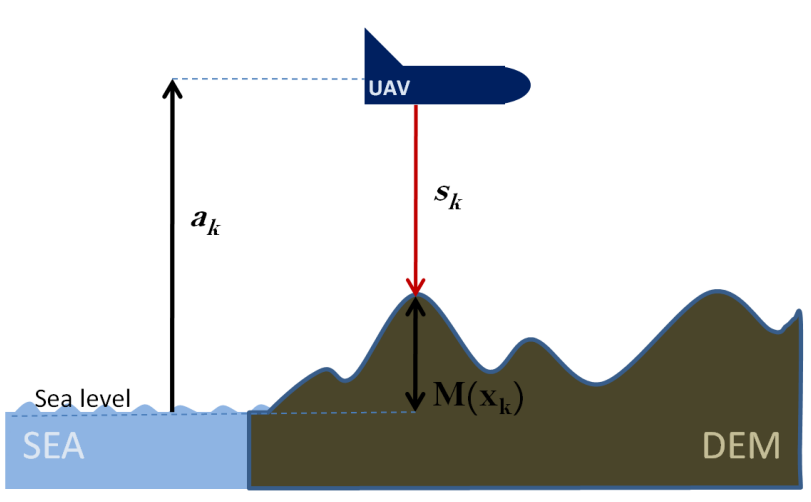

Fig. 1. Echo radar TBN diagram for UAV

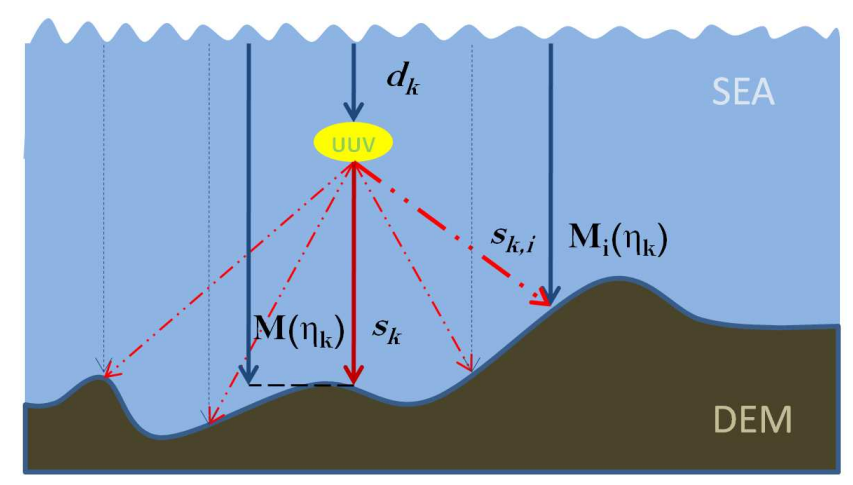

Fig. 2. Multi beam sonar TBN diagram for UUV 


\subsection{Sonar Profile to Map Correlation}

An alternative method to formulate the observation equation consist on correlating the measured profile $z_{t}$ to the stored elevation map $\mathbb{M}$ to evaluate a degree of similarity of each candidate pose $\mathbf{x}_{t}$ within the map domain, and select the one which maximizes the correlation. Several methods have been used in the literature to compute the correlation: 1) the cross-correlation (COR), 2) The Absolute Square Distance (ASD), 3) the Mean Absolute Difference (MAD) and 4) the Minimum Square Distance (MSD):

$$
\begin{gathered}
C O R\left(\mathbf{x}_{t}\right)=1 / N \sum_{i=1}^{N}\left(z_{t, i}-h_{i}\left(\mathbf{x}_{t}\right)\right) \\
A S D\left(\mathbf{x}_{t}\right)=\sum_{i=1}^{N}\left(z_{t, i}-h_{i}\left(\mathbf{x}_{t}\right)\right)^{2} \\
M S D\left(\mathbf{x}_{t}\right)=1 / N \sum_{i=1}^{N}\left(z_{t, i}-h_{i}\left(\mathbf{x}_{t}\right)\right)^{2} \\
M A D\left(\mathbf{x}_{t}\right)=1 / N \sum_{i=1}^{N}\left|\left(z_{t, i}-h_{i}\left(\mathbf{x}_{t}\right)\right)\right|
\end{gathered}
$$

These methods are $O\left(n^{2}\right)$, since they explore the complete map. Hence, some research has been carry out to accelerate the process. Baker and Clem (1977) use a dead reckoning estimate to bound the area where the correlation is computed and Ingemar Nygren (2003) has designed a dedicated high speed hardware based on FPGAs to face the problem. When correlation is used, a simple linear observation equation is need where $z_{t}=\underset{\mathbf{x}_{t}}{\operatorname{argmax}}\left\{C O R\left(\mathbf{x}_{t}\right)\right\}$.

\section{THE SEMINAL WORK OF TERCOM}

TBN can be traced back to 50 years ago, when terrain contour matching (TERCOM) was successfully developed as a navigation method for cruise missiles. TERCOM evolved after some years, in many similar approaches (LACOM, RACOM, SAMSOM, ... (see Siouris (2004))). TERCOM is a batch oriented algorithm which periodically correlates the measured bottom profile with the elevation map (see Figure 3). The algorithm operates in three phases. First, digital maps of the terrain to be traversed are selected. At this step, is crucial to select a terrain with a profile rich enough. This can achieve by means of computing the terrain roughness (see Equation 12), as the standart deviation of the terrain function $\mathbb{M}\left(\eta_{i}\right)$ with respect to the desired flight altitude $a_{i}$ (Figure 1 ):

$$
\sigma=\sqrt{1 / N \sum_{i=1}^{N}\left(\mathbb{M}\left(\eta_{i}\right)-a_{i}\right)^{2}}
$$

Then, the path with highest standard deviation of the terrain function is selected. Periodically, during the flight, at intervals related to the size cells, the altitude with respect to the bottom is acquired. During this phase, the aircraft is not allowed to maneuver. Finally, at the last step, the patches of the digital map are correlated with the profile acquired by the altimeter using the Mean Absolute Difference (MAD).

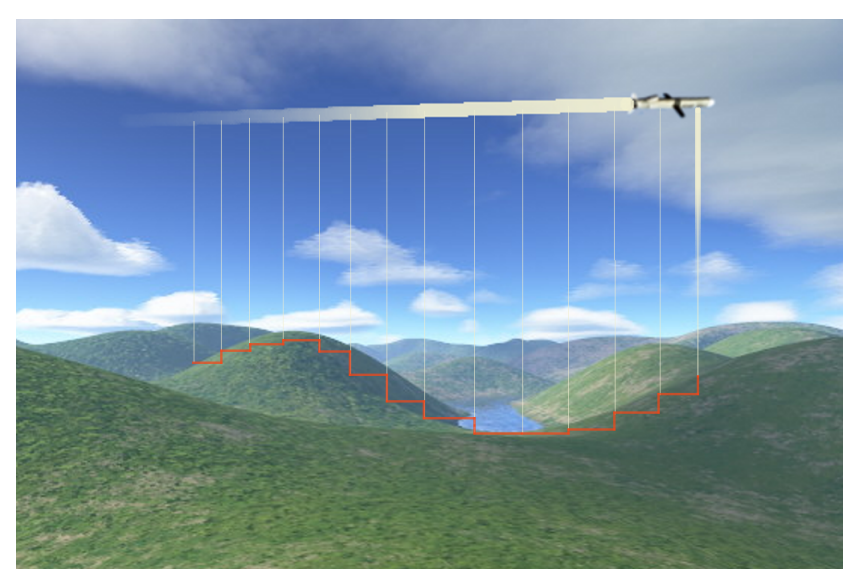

Fig. 3. The principle of Terrain Based Navigation.

\section{BAYESIAN ESTIMATION TECHNIQUES FOR TBN}

Let us assume $\mathbf{x}_{t}$ to be a Markovian state and

- $p\left(\mathbf{x}_{t-1}\right)$ be the probability density function (pdf) describing the probability of the robot of being at a certain pose at time $t-1$,

- $p\left(\mathbf{x}_{t} \mid \mathbf{x}_{t-1}, u_{t}\right)$ be the state transition probability, also known as motion model, which allows to predict the robot pose after certain input $\mathbf{u}_{t}$,

- $p\left(\mathbf{z}_{t} \mid \mathbf{x}_{t}\right)$ be the measurement probability which given a map $\mathrm{M}(\mathbf{x})$ provides the probability of observing $\boldsymbol{z}_{\boldsymbol{t}}$ when being at state $\mathbf{x}_{t}$,

then, the TBN problem consists on solving for $p\left(\mathbf{x}_{t}\right)$, given $p\left(\mathbf{x}_{t-1}\right), p\left(\mathbf{x}_{t} \mid \mathbf{x}_{t-1}, \mathbf{u}_{t}\right)$ and $p\left(\mathbf{z}_{t} \mid \mathbf{x}_{t}\right)$. In the context of bayesian estimation this can be done through the Bayes filter $(\mathrm{BF})$ :

$$
\begin{gathered}
p\left(\overline{\mathbf{x}}_{t}\right)=\int p\left(\mathbf{x}_{t} \mid \mathbf{x}_{t-1}, \mathbf{u}_{t}\right) p\left(\mathbf{x}_{t-1}\right) d \mathbf{x}_{t} \\
p\left(\mathbf{x}_{t}\right)=\eta p\left(\mathbf{z}_{t} \mid \mathbf{x}_{t}\right) p\left(\overline{\mathbf{x}_{\mathbf{t}}}\right)
\end{gathered}
$$

In the most general case, the BF cannot be implemented because it relies on the close form solution of the integral shown in (13). Under some conditions, the BF can be implemented using parametric filters like the Kalman Filter (KF), the Information Filter (IF) or their nonlinear counter parts like the Extended Kalman Filter (EKF), the Extended Information Filter (EIF) or the Unscented Kalman Filter (UKF); or non parametrics filters like the Point Mass Filter (PMF), the Particle Filter (PF) or the Rao-blackwellised Particle Filter (RBPF) (see Thrun (2002)). During the last years, these probabilistic approaches have become dominant in TBN applications. The popularity of those Bayesian estimation techniques has grown because these probabilistic algorithms explicitly deal with the uncertainties in both, the motion and the measurements models. It is well known that these uncertainties play an important role to solve the robot navigation and mapping problems. In the following subsections we review the main works using bayesian estimation techinques for TBN. 


\section{$4.1 \mathrm{KF}$ based Solutions to the TBN}

It is well known that the Bayes Filter (BF) can be optimally implemented as a Kalman Filter (KF) when the following conditions hold:

- The probability density function describing the robot position $p\left(x_{t}\right)$ and the measurement probability $p\left(z_{t} \mid x_{t}\right)$ are both Gaussian.

- The state transition probability $p\left(x_{t} \mid x_{t-1}, u_{t}\right)$ can be represented by means of a linear stochastic equation.

- The measurement models are driven by mutually uncorrelated zero mean white noise sequences.

Some authors like Nygren and Jansson (2004) and Bergem (1993) have proposed the use of a KF to estimate the position posterior. The vector state contains the robot position referenced to the earth fixed frame $\mathbb{E}$ (see Nygren and Jansson (2004)) and sometimes includes also the robot velocity and even the acceleration, both referenced to the $\mathbb{E}$-frame (see Bergem (1993)). Depending on the available sensors for dead reckoning, different methods have been proposed for motion prediction. Nygren and Jansson (2004) used an INS for estimating the robot displacement and performed a reset after each motion step. Bergem (1993) studied different motion models including: 1) constant velocity, 2) constant acceleration, 3) constant acceleration with maneuvering detection to increase/decrease the uncertainty of the prediction, and 4) the use of colored noise for modeling the exponential auto-correlated accelerations due to the robot maneuvers.

The most used sensor to perceive the terrain is the multibeam echo-sounder. The measured terrain profiles are correlated to the stored map to find the robot pose to update the filter by means of a linear measurement model. Nygren and Jansson (2004) proposed to use the Absolute Square Distance (ASD) (see table 1) for profile to map correlation. Under the assumption of large time between measurements, the author argues that the position prior has a large variance and hence the problem becomes a ML-estimation problem. For this case, the asymptotic covariance matrix is known to be the inverse of the fisher information matrix which can be computed as the Hessian of the likelihood function. An alternative method is proposed in Bergem (1993) who proposed to use a matching strength function ( $f$ ) based on an Exponential Normalized Squared Absolute Distance (ENSAD) for profile to map correlation. First, a validation gate is defined over the Mahalanobis distance of the position innovation. It defines a region where the candidate measurements statistically compatible with the predicted measurement should lay. The position maximizing the $f$-function within this region is chosen as the measurement. Next a grid of certain resolution is defined within the validation region and the $f$-function is evaluated so the Relative Measurement Covariance Matrix (RMCM) is computed to be used later for the KF update. A different approach was used by Di Massa (1997) who proposed to use a Probability Data Association Filter with amplitude information (PDAFAI), a variation of the Probability Data Association Filter (PDAF) rooted in the KF framework. In this case, the robot velocity is used as a control input of a linear motion model to predict the robot position. Again a validation gate is defined where the sonar profile is matched against the map using the MAD criterion obtaining a list of matching candidates each one with a certain probability of being the correct one. As a difference with respect to a conventional KF which would probably use the nearest neighbor criteria to select one of them, the PDAF combines all of them, through a probabilistic weight average, into a unique matching candidate which is then used for the update. While in PDAF the MAD criteria is only used for validating the measurements, in PDAFAI the MAD is also used to evaluate the matching probability of each measurement.

When the motion and/or the measurement model are nonlinear, an Extended Kalman Filter (EKF) should be used. The EKF uses a first order Taylor expansion to approximate the nonlinear functions. The accuracy of such expansion depends on the nonlinearity of the system, and the width of the posterior. EKF tend to obtain good results if the state of the system is known with relatively high accuracy, so that the remaining covariance is small. The larger the uncertainty the higher the error introduced by the linearization.

In the feature based map matching method presented by Sistiaga et al. (1998), the common features founded in the observed map and the stored one, are matched and used to find the best transformation between them, thus this information is used as a measurement to refine the heading and position of the vehicle using an EKF.

\subsection{Multi Model Adaptative Estimation Techniques}

EKFs are known to work well when the uncertainty of the estimate is small. When the uncertainty is very large the linear Gaussian approximation fails and the filter diverges. For this reason several authors have proposed to use Multi Model Adaptative Estimator (MMAE) techniques following a classical "Divide and conquer" approach. Instead of using a unique estimate with a large uncertainty, MMAE techniques used a cloud of estimates, each one with its own uncertainty to cover the large uncertain region. Hostetler and Andreas (1983) used a bank of KFs initialized at positions biased with respect to the INS estimate. The filter for which the Average Weighted Residual Squared (AWRS) between the predicted ground clearance and the ground clearance measured by the altimeter is chosen as the navigation output.

Similarly Boozer et al. (1985), proposed the Sandia Inertial Terrain-Aided Navigation (SITAN) where a bank of three error state KFs is used to estimate the north, east and vertical channel biases of the INS. SITAN distributes uniformly the set of KFs covering the area of uncertainty of the current INS estimate. If a set of fix decision rules are satisfied, the bias estimates from the best candidate filter in the bank are added to the reference navigation systems position to form an estimate of the aircraft's current position. Otherwise, the system is reset to the initial uniform distribution, and the process starts again. This algorithm was later adapted to helicopters becoming Heli/SITAN Hollowell (1990) which uses a bank of one error estate KFs to estimate the vertical channel bias. In this case, each EKF represent fixed $\mathrm{x}-\mathrm{y}$ bias around the INS estimate. Hence, each static KF uses the same measured altitude, but compared against a different region of the elevation map. In order to estimate the true position bias, the 3 
by 3 neighborhood cells around the best candidate are conveniently merged into a unique bias estimate to be used to reset the INS. Other authors Jianchun et al. (2007) have recently used the same approach. In Oliveira (2007) a MMAE method together with a PCA based navigation algorithm has been proposed. In this case, the navigation output is a probabilistic weigh average of the set of estimates based on the filter residuals, as an application of the seminal work of Anderson et al. (1979).

\subsection{Solutions to the TBN}

PFs, also called sequential Monte-Carlo (SMC) methods, are recursive filters for solving the Bayesian estimation problem which can deal with nonlinear motion and/or measurement models without relying on linearization techniques. PFs use a point mass representation of the density function to approximate the robot pose posterior. Hence a set of random samples, "particles", are used to represent the probability density. This non parametric representation of a pdf is able to represent a much broader space of distributions than Gaussians, like for instance multi modal pdfs. A Similar approach to the PF is the Point Mass Filter (PMF) presented by Doucet et al. (2000). The PMF computes a discrete approximation of the probability density function of the vehicle position and recursively updates it with each new measurement from the mapping and navigation sensors. While PF represent the probability according to the density of the particles laying in a certain region, in PMF the particles are distributed according a static mesh, representing the probability through the weight of the particles.

In order to improve the accuracy, the grid mesh resolution is dynamically adapted. Low weight particles are deleted and when the number of particles is bellow a threshold, the mesh resolution is increased duplicating the current set of particles. Anonsen and Hallingstad (2006) compared the PMF against the PF achieving slightly better results for the PMF, but at a higher computational cost. For high dimensional spaces this cost is prohibitive for real time applications therefore, the same author prosposed to use submaps to overcome this problem.

The SIR Particle Filter. A widely used variant of the $\mathrm{PF}$ is the Sampling Importance Resampling PF (SIR-PF) (see Gordon et al. (1993)). The Sequential Information Sampling PF (SIS-PF) is known to suffer from a strong degeneracy problem where after a while all but one particle will have negligible weight. To reduce this effect the SIR filter introduces a resampling step to eliminate particles with small weight while duplicating particles with high weight. A SIR-PF TBN method using an INS for measuring the robot displacement and an echo sounder for measuring the altitude has been presented in Karlsson et al. (2003). This work was later extender to surface vessels using radar measurements (Anonsen and Hallingstad (2006)). In their work the authors carry out an analysis of the navigation performance based on the study of Cramer Rao Lower Bound (CRLB), presenting an analytical expression under the stationary assumption. Through Monte Carlo simulation but using a real map, authors conclude that it is possible to achieve a RMSE of the SIR-PF TBN system close to the CRLB but at the cost of using a high number of particles $(>10000)$.

In (Maurelli et al. (2008)) a SIR-PF TBN system is proposed where the robot displacement is measured with a FOG-DVL navigation system and the range-altitude as well as range profiles acquired with a Mechanical Scanning Profiling Sonar are used to sense the terrain. Authors propose to inject random particles during the resampling process in order to allow the system to recover from possible wrong convergence situations. A similar method was formerly described in the Augmented-MCL algorithm of Thrun (2002). The proposed system has been tested in $3 \mathrm{D}$ through simulation, and in $2 \mathrm{D}$ in water tank as well as in a Marina Environment (Maurelli et al. (2009)), succesfully solving the TBN problem in those environments.

The Rao Blackwellised Particle Filter. The PF solution to the TBN which relies on solving for posterior $p\left(\mathbf{x}_{0: t} \mid z_{1: t}\right)$ through sampling based methods, needs a very large number of particles to deal with high dimensional state spaces of $\mathbf{x}$. When the state variable can be divided into two groups $\mathbf{x}_{0: t}=\left[\mathbf{x}_{0: t}^{\prime}, \mathbf{x}_{0: t}^{\prime \prime}\right]^{T}$ and $p\left(\mathbf{x}_{0: t}^{\prime} \mid \mathbf{z}_{1: t}, \mathbf{x}_{0: t}^{\prime \prime}\right)$ is analytically tractable it is possible to use a Rao-Blackwellised Particle Filters (RBPF) (Doucet et al. (2000)) using the chain rule to decompose the posterior:

$$
p\left(\mathbf{x}_{0: t}^{\prime}, \mathbf{x}_{0: t}^{\prime \prime} \mid \mathbf{z}_{1: t}\right)=p\left(\mathbf{x}_{0: t}^{\prime} \mid \mathbf{z}_{1: t}, \mathbf{x}_{0: t}^{\prime \prime}\right) p\left(\mathbf{x}_{0: t}^{\prime \prime} \mid \mathbf{z}_{1: t}\right)
$$

A common case appears when $p\left(\mathbf{x}_{0: t}^{\prime} \mid \mathbf{z}_{1: t}, \mathbf{x}_{0: t}^{\prime \prime}\right)$ is a linearGaussian system. In this case each particle includes a KF (mean \& covariance) to estimate $\mathbf{x}^{\prime}$ (from now on $\mathbf{x}^{k f}$ ) and a sample representation of $\mathbf{x}^{\prime \prime}$ (from now on $\mathbf{x}^{p f}$ ) - In this way RBPF reduces the dimensionality of the problem being able to achieve faster and more accurate results. For an aerial vehicle, and describing the altitude by means of a KF, Nordlund and Gustafsson (2002) have used a RBPF in a simulated TBN application. The RBPF has been compared with a standard SIR-PF, using the same three dimensional state vector. As expected, authors show that the number of particles needed to estimate the position is larger when the PF is used. Surprisingly the accuracy of the RBPF is worse than the accuracy of the $\mathrm{PF}$, fact that the authors attribute to a wrong modeling of the altitude noise. The radar sometimes observes the top of the trees and some times the floor, being the noise a bi-modal distribution. In underwater applications Teixeira (2007) used a RBPF to merge measurements of range to the bottom (sonar altimeter, forward looking echo sounder, and side looking echo sounder) with deadreckoning data (DVL+MRU). In their approach the state vector contains the $2 \mathrm{D}$ robot position and a $2 \mathrm{D}$ velocity bias due to unknown ocean currents. A simple kinematics model using velocity, heading and depth inputs is used together with mutually independent Gaussian white process noise. Due to the linear nature of the bias model, a rao-blackwellization process allows to estimate the robot pose using a PF while estimating the velocity bias using a KF. The two main contribution of Teixeira consist on proposing the Smoothed Kernel Particle Filter (SKPF) to obtain more consistent results and an improved robustness to outliers and the complementary use of Geophysical Navigation to improve the navigation results in flat terrains. 


\section{SUMMARY}

A summary of the most representative techniques is grouped by filtering technique in (Table 1). In order to produce a useful comparative, the same nomenclature has been used, and is presented below.

\section{CONCLUSION}

This paper has introduced the TBN problem. To the best of the author's knowledge, the most representative works in the field have been described and classified. To allow for a more simple comparative, a table with an unified nomenclature has been presented, detailing: the principal sensor, the state and the input vectors, the measurement, the motion and the measurement models, with the aim of highlighting the fundamental similarities as well as to strength their main singularities.

\section{REFERENCES}

Anderson, B., Moore, J., and Barratt, J. (1979). Optimal filtering. Prentice-Hall Englewood Cliffs, NJ.

Anonsen, K. and Hallingstad, O. (2006). Terrain aided underwater navigation using point mass and particle filters. In Proceedings of the IEEE/ION Position Location and Navigation Symposium.

Baker, W. and Clem, R. (1977). Terrain contour matching [TERCOM] primer. Technical ReportASP-TR-77-61. AeronauticalSystems Division, Wright-Patterson AFB.

Bergem, O. (1993). Bathymetric navigation of autonomous underwater vehicles using a multibeam sonar and a Kalman filter with relative measurement covariance matrices. Dr. Scient thesis, University of Trondheim, Norway.

Bergman, N., Ljung, L., and Gustafsson, F. (1999). Terrain navigation using Bayesian statistics. IEEE Control Systems Magazine, 19(3), 33-40.

Boozer, D., Lau, M., and Fellerhoff, J. (1985). The AFTI/F 16 terrain-aided navigation system. In National Aerospace and Electronic Conference.

Di Massa, D. (1997). Terrain-relative navigation for autonomous underwater vehicles. Ph.D. thesis, Massachusetts Institute of Technology.

Doucet, A., Godsill, S., and Andrieu, C. (2000). On sequential Monte Carlo sampling methods for Bayesian filtering. Statistics and computing, 10(3), 197-208.

Durrant-Whyte, H. and Bailey, T. (2006). Simultaneous localization and mapping: part I. IEEE Robotics $\&$ Automation Magazine, 13(2), 99-110.

Fossen, T.I. (2002). Marine Control Systems: Guidance, Navigation and Control of Ships, Rigs and Underwater Vehicles. Marine Cybernetics AS, Trondheim, Norway, 1st edition.

Gordon, N., Salmond, D., and Smith, A. (1993). Novel approach to nonlinear/non-Gaussian Bayesian state estimation. In IEE Proceedings, volume 140, 107-113.

Hollowell, J. (1990). Heli/SITAN: A terrain referenced navigation algorithm for helicopters. PLANS 90; 20-23 Mar 1990.

Hostetler, L. and Andreas, R. (1983). Nonlinear Kalman filtering techniques for terrain-aided navigation. IEEE Transactions on Automatic Control, 28(3), 315-323.
Ingemar Nygren, M.J. (2003). Terrain Navigation for Underwater Vehicles Using the Correlator Method. Journal of Oceanic Engineering.

Jianchun, X., Rongchun, Z., and Yong, X. (2007). Combined Terrain Aided Navigation based on Correlation Method and Parallel Kalman Filters. In Electronic Measurement and Instruments, 200\%. ICEMI'0\%. 8th International Conference on, 1-145.

Karlsson, R., Gustafsson, F., and Karlsson, T. (2003). Particle filtering and Cramer-Rao lower bound for underwater navigation. In 2003 International Conference on Acoustics, Speech, and Signal Processing (ICASSP'03).

Mallios, A., Ribas, D., and Ridao, P. (2009). Localization Advances in the Unstructured Underwater Environment. Proceedings of the 9th Hellenic Symposium of Oceanography and Fishery, 1, 111 - 116.

Maurelli, F., Krupinski, S., Petillot, Y., and Salvi, J. (2008). A Particle Filter Approach for AUV Localization.

Maurelli, F., Mallios, A., Ribas, D., Ridao, P., and Petillot, Y. (2009). Particle Filter Based AUV Localization using Imaging Sonar. IFAC $M C M C$.

Nordlund, P. and Gustafsson, F. (2002). Recursive estimation of three-dimensional aircraft position using terrainaided positioning. In IEEE International conference on acoustics speech and signal porcessing, volume 2. IEEE; 1999.

Nygren, I. and Jansson, M. (2004). Terrain navigation using the correlator method. In Position Location and Navigation Symposium, 2004. PLANS 2004, 649-657.

Oliveira, P. (2007). MMAE terrain reference navigation for underwater vehicles using PCA. International Journal of Control, 80(7), 1008-1017.

Siouris, G. (2004). Missile guidance and control systems. Springer Verlag.

Sistiaga, M., Opderbecke, J., Aldon, M., and Rigaud, V. (1998). Map based underwater navigation using a multibeam echosounder. In OCEANS'98 Conference Proceedings, volume 2.

Teixeira, F. (2007). Terrain-Aided Navigation and Geophysical Navigation of Autonomous Underwater Vehicles. Ph.D. thesis, Instituto Superior Tcnico, Universidade Tcnica de Lisboa.

Thrun, S. (2002). Probabilistic robotics, volume 45. ACM. 


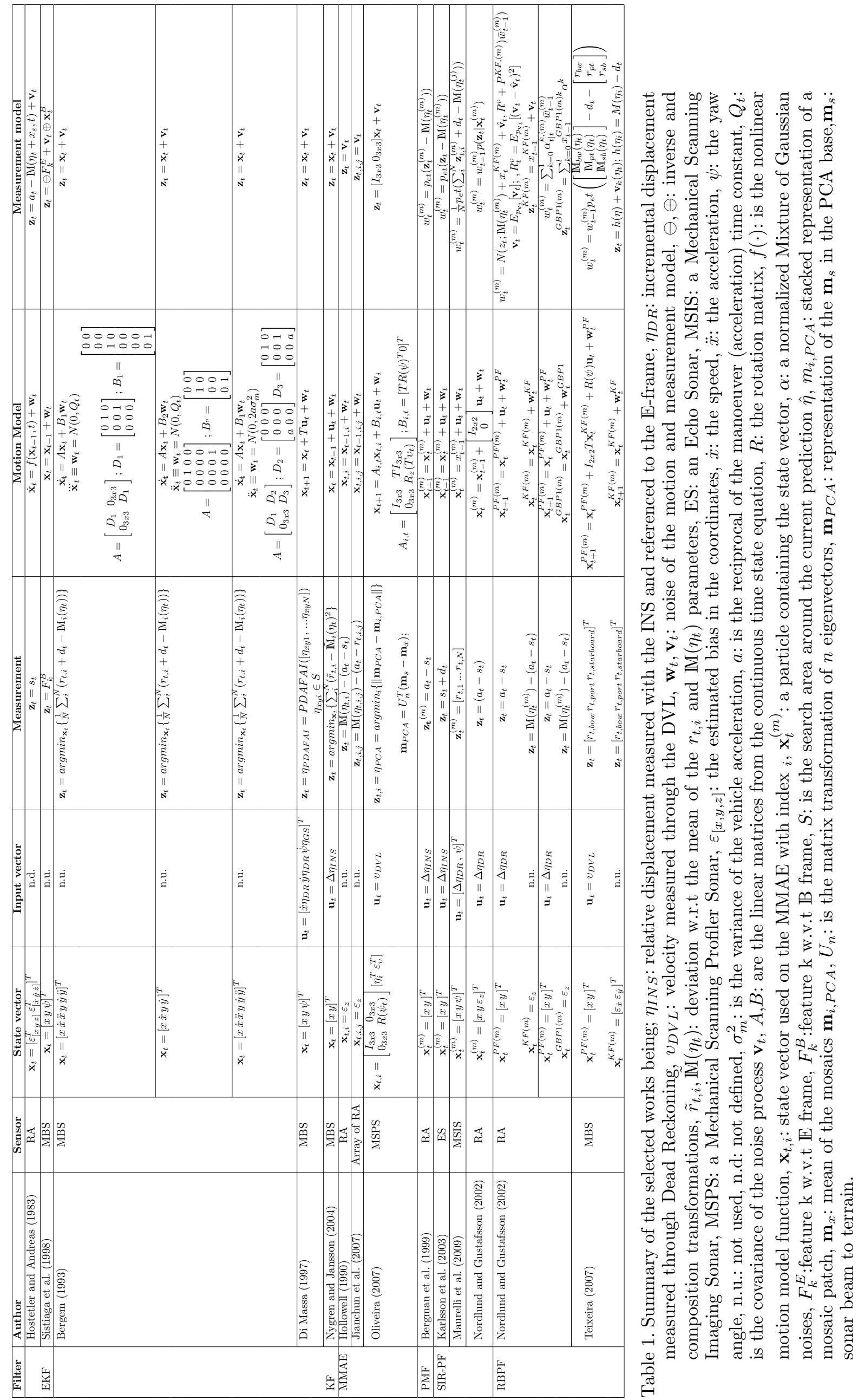

